\title{
Level Set Method for Image Segmentation: A Survey
}

\author{
Vineetha G R ${ }^{1}$, Gopu Darshan ${ }^{2}$ \\ ${ }^{1,2}$ (Computer science Department, Sree Buddha College of Engineering /Kerala University, India
}

\begin{abstract}
A considerable challenge in image segmentation in real world images is intensity inhomogenity. The existing image segmentation algorithms rely on region based and it typically features similarity of image intensities in the region of interest. Due to the presence of intensity non uniformity the segmentation results are not accurate. So this survey paper gives a novel region based techniques for image segmentation in the presence of intensity inhomogenity. Intensity non uniformity occurs in real world images due to various factors such as spatial variations on illumination, imperfections of imaging devices, noise and of low contrast, causes many problems in image processing and computer vision. Image segmentation is a very difficult task in the presence of non uniformity of intensities. Intensity non uniformity is caused by the overlaps between the ranges of intensities in the region to be segmented. This paper covers segmentation techniques to overcome the intensity inhomogenity with the help of Level Set Method. The aim is to implement a level set approach for active contour image segmentation for MRI images. No requirement about object's shape and allowances for very flexible topology change are key advantages for this method. As an application our method can be used for segmentation and bias correction of magnetic resonance images with accurate results.
\end{abstract}

Keywords - Contour, Image Segmentation, Intensity Inhomogenity, and Level set Method, MRI images

\section{Introduction}

In computer vision, the process of partitioning a digital image into multiple segments is image segmentation. To simplify and or change the representation of an image into something that is more meaningful and easier to analyze is the goal of segmentation. The main uses of Image segmentation are to locate objects and boundaries (lines, curves, etc.) in images. The process behind image segmentation is to assign a label to every pixel in an image such that pixels with the same label share certain visual characteristics. Segmentation techniques can be stated as the methods that are used for extracting and representing the information from an image. The accuracy of segmentation is determined by the essential success or failure of computerized analysis procedure. A set of segments that collectively cover the entire image, or a set of contours extracted from the image is the result of image segmentation. Each of the pixels in a region are similar with respect to some characteristics or computed property such as color, intensity or texture. When applied to a stack of images, typical in medical imaging, the resulting contours after image segmentation can be used to create 3D reconstructions with the help of interpolation algorithms like marching cubes. There are so many applications for image segmentation .The main practical applications include content based image retrieval, Machine vision, Medical Imaging, Object detection, Pedestrian detection, Face detection, Brake light detection, Locate objects in satellite images, Recognition Tasks, Iris recognition, Traffic control systems. The main applications of medical imaging are Locate tumors and other pathologies, Measure tissue volumes, Diagnosis \& study of anatomical structure.

Segmentation is process of subdividing an image into its constituent regions or objects. This segmentation is mainly done of one of two basic properties of intensity values. The properties are discontinuity and similarity. Similarity (homogeneity) in images is to partition an image into regions that are similar according to a set of predefined criteria in images. Homogeneity refers to the uniformity (similarity) of images. Discontinuity property can be stated as partition of an image is based upon sharp changes in intensity. The similarity of pixels mainly deals with the homogeneity of intensity in the image. Most of the existing segmentation methods rely on the segmentation of homogeneous images. Presence of Intensity In homogeneities in images is hard to process. With the help of level set method we can do segmentation in the presence of intensity inhomogenity. Level Set Method is a numerical technique for tracking interfaces and shapes. The main advantages of level set method are that one can perform numerical computations involving curves and surfaces on a fixed Cartesian grid without having to parameterize these objects. The method is implemented by partial differential equation in particular Hamilton -Jacobi equation and can be solved numerically by using finite differences on a Cartesian grid.

This paper is organized as follows. In Section 2 various aspects image segmentation is discussed. Both region-based and edge-based approaches to image segmentation are described. Section 3 the Level Set Method for Image Segmentation is explained. Section 4 an application of image segmentation in the field of medical 
image processing. Section 5 gives a summary regarding various level set methods applied to image segmentation Section 6 describes the conclusion.

\section{Image Segmentation Methodology}

Image segmentation, as mentioned above is widely used in content based image retrieval, Machine vision, Medical Imaging ,Object detection, Pedestrian detection, Face detection, Brake light detection, Locate objects in satellite images, Recognition Tasks, Iris recognition, Traffic control systems. The main applications of medical imaging are Locate tumors and other pathologies, Measure tissue volumes, Diagnosis \& study of anatomical structure. Medical imaging which consists mainly combination of sensors recording the anatomical body structure like magnetic resonance image (MRI), ultrasound or CT with sensors monitoring functional and metabolic body activities like positron emission tomography (PET), single photon emission computed tomography (SPECT) or magnetic resonance spectroscopy (MRS). Results can be applied, for instance, in radiotherapy and nuclear medicine. This paper mainly deals with the application on magnetic resonance image. An image segmentation can be described as Let the image domain be $\Omega$ and $\mathrm{P}_{\mathrm{i}}$ be the partitions of $\Omega$ Such that $\mathrm{P}_{\mathrm{i} \subset} \Omega, \Omega=\cup_{i=1}^{n} \mathrm{P}_{\mathrm{i}}, \mathrm{H}\left(\mathrm{P}_{\mathrm{i}}=\right.$ true $\forall \mathrm{mH}\left(\mathrm{P}_{\mathrm{i}} \cup \mathrm{P}_{\mathrm{j}}\right)=$ false $\forall \mathrm{P}_{\mathrm{i}}$ and $\mathrm{P}_{\mathrm{j}}$ adjacent Where $\mathrm{P}_{\mathrm{i} \cap} \mathrm{P}_{\mathrm{j}}=\phi$ for $\mathrm{i} \neq \mathrm{j}$ and each $P_{i}$ is connected [1]If an image has been pre-processed appropriately to remove noise and artifacts, segmentation is often the key step in interpreting the image. Image segmentation is a process in which regions or features sharing similar characteristics are identified and grouped together. Image segmentation may use statistical classification, thresholding, edge detection, region detection, or any combination of these techniques. The output of the segmentation step is usually a set of classified elements.

Most segmentation techniques are either region-based or edge based- Region-based techniques rely on common patterns in intensity values within a cluster of neighboring pixels. The cluster is referred to as the region, and the goal of the segmentation algorithm is to group regions according to their anatomical or functional roles. Edge-based techniques rely on discontinuities in image values between distinct regions, and the goal of the segmentation algorithm is to accurately demarcate the boundary separating these regions. Segmentation is a process of extracting and representing information from an image is to group pixels together into regions of similarity. The categories of image segmentation mainly include Clustering Methods - Level Set Methods, Histogram-Based Methods- Graph Partitioning Methods, Edge Detection Methods- Watershed Transformation, Region Growing Methods- Neural Networks Segmentation. In this paper we mainly go through the clustering methods which include Level Set Method

\section{LEVEL SET METHOD}

The method was implemented by Osher and Sethian; mainly rely on the theory of curve and surface evolution and on the link between front propagation and hyperbolic conservation laws. The level Set Method is a numerical technique for tracking interfaces and shapes [2] has been increasingly applied to image segmentation. This method makes it easy to follow shapes that change topology, means the shape can be split into two for developing holes and the reverse of these operations is also possible. In this method contours or surfaces are represented as a zero level set of a higher dimensional function, usually called a level set function. The image segmentation problem can be formulated and solved in a principled way based on well established mathematical theories including calculus of variations and partial differential equations can be achieved with the help of level set representation. Level set approach is numerically most stable implicit representation. The method is acting as a great tool for modeling time varying objects. There are two types of level set formulations which include Time dependent level set formulation and Stationary level set formulation. Two important numerical issues regarding level set method is Level Set Method does not implicitly preserve the level set function as a distance function and the algorithm is slow because the time step is limited by the CFL condition. The main advantage of Level Set Method is that one can perform numerical computations involving curves and surfaces on a fixed Cartesian grid without having to parameterize these objects. The level set method amounts to representing a closed curve using auxiliary function called level set function which is represented as the zero level set. The level set method defines problem in one higher dimension. The zero level set at one point in time as a slice of the level set surface. The formulation of level set implies that the level set value of a point on the contour with motion must always be zero. The level set method is boundary driven and region driven model free segmentation

\section{Medical Image Processing}

An important application of image segmentation is in the field of medical image processing. The technique and process used to create the images of human body is medical imaging. The main applications of medical imaging are used for clinical purposes. Imaging technology in medicine made the doctors to see the interior portions of the body for easy diagnosis. It also helps doctors to make keyhole surgeries for reaching the 
interior parts without really opening too much of the body. Medical imaging constitutes a sub-discipline of biomedical engineering, medical physics or medicine depending on the context. Many of the techniques developed for medical imaging also have scientific and industrial applications. Medical imaging is often perceived to designate the set of techniques that noninvasively produce images of the internal aspect of the body. In this restricted sense, medical imaging can be seen as the solution of mathematical inverse problems. This means that cause is inferred from effect. The term non-invasive is a term based on the fact that following medical imaging modalities do not penetrate the skin physically. But on the electromagnetic and radiation level, they are quite invasive. From the high energy photons in X-Ray Computed Tomography, to the 2+ Tesla coils of an MRI device, these modalities alter the physical and chemical environment of the body in order to obtain data. There are so many imaging techniques are available. Magnetic Resonance Imaging took over x-ray imaging by making the doctors to look at the body's elusive third dimension. Quantitative means of analyzing Multidimensional image data is MRI. MRI traditionally creates a two dimensional image of a thin "slice" of the body and is therefore considered a tomography imaging technique. Modern MRI instruments are capable of producing images in the form of 3D blocks, which may be considered a generalization of the single-slice, tomography concept. Unlike CT, MRI does not involve the use of ionizing radiation and is therefore not associated with the health hazards. MRI provides high quality images of the inside parts of the human body. An MRI machine uses a magnetic field and radio waves to create detailed images of the body The advantage of using MRI is no ionizing radiation is involved.

\section{Literature Survey \\ 5.1 A Novel Shape Prior Based Level Set Method for Liver Segmentation From MR Images [3] .}

This paper propose a novel level set based variational approach that incorporates shape prior knowledge into the Chan Vese model which can overcome the leakage and over segmentation problems. Statistical methods are used to get the prior shape and the training process allows the prior shape not exactly at the location of desired object. But all these models above Fail to segment object from images where the objects are occluded by other objects or some parts of them are in low gray contrast or missing because they are all gray intensity based. These methods do not allow for translation, rotation, and scaling of the prior shape. The proposed method allows translation, rotation and scaling of the prior shapes and also performs object supervision before segmentation to achieve better result and higher performance. This is mainly achieved with the help of affine transformation. The proposed method novel level set base variational model for segmentation using prior shapes which helps us to detect the liver perfusion position and measure the intensity. This helps to achieve faster speed and we propose a new measure which help to perform the training of given shapes. If some part of the image is occluded or missing, we can still get a reliable segmentation result

\subsection{Variational Image Segmentation Models: Application to medical images MRI [4]}

An important branch of computer vision is image segmentation. The image segmentation problem can be solved with the help of mathematical frame work based on variational model and partial differential equations. This framework is defined in a continuous setting which makes the proposed model independent with respect to the grid of digital images This paper mainly introduces a segmentation method based on Variational approach. These models are defined in a continuous setting and are mathematically well established. Two well known image segmentation based on variational approach is specified. They are Mumford Shah model and level set approach. The Mumford Shah model is mainly used to minimize the energy function. The level set method was introduced for tracking moving fronts. The theory of curve/surface convolution methods are used here which efficiently solve the problem of moving fronts especially in the problem of change of topology. In traditional level set methods it is necessary to initialize the level set method function as a signed distance function. But the proposed method suggests the following function as the initial function. Hence this method is more suitable than other existing segmentation methods.

\subsection{A Variational Multi Phase Level Set Approach To Simultaneous Segmentation And Bias Correction [5]}

This paper represents a novel level set approach to simultaneous tissue segmentation and bias correction of MRI.The intensity of each tissue is modeled as a Gaussian distribution of spatially varying mean and variance. The sliding window is used to transform intensity from one dimension to another. The objective function is distributed over each point and integrated over the entire domain to form a variational level set evolution. With the help of level set evolution process tissue segmentation and bias correction are achieved. The proposed method uses a variational level set approach to simultaneous segmentation and bias correction. The method uses a k-Means clustering which is weighted k-means variational level set. The proposed method use a special case SVMLS-Statistical \& variational multiphase level set. Advantage of this method is that the smoothness of the computed bias field is ensured by the normalized convolution without extra cost. 


\subsection{Model-Based Medical Image Segmentation: A Level Set Approach [6]}

This method mainly requires the definition of a speed function that governs model deformation. This mainly considers the region intensity information while the existing methods usually do image gradient information. The region intensity information into the level set framework forms an accurate and robust segmentation. The level set approach the convergence to the final result may be relatively independent of the initial shape, and branches and splits and merges can develop without problems as the front moves. The challenges in level set approach are to construct an adequate model for the speed function. The main idea is to define a range of intensity values that classify the tissue type of interest and then base the propagation term on the level set equation for that intensity range. Using this approach the smoothness of the evolving surface can be used to prevent the leakage which is common in connected component schemes.

\subsection{A Variational Level Set SAR Image Segmentation Approach Based on Statistical Model [7].}

Due to the presence of speckle noise SAR image automatic segmentation is difficult task. A variational level set approach for SAR image is presented in this paper which states a new energy functional is defined by taking account of a statistical model of speckle noise. The energy functional produced in this paper is different from the energy functional with respect to the parameterized curve in general level set approach. By minimizing the energy functional in level set approach segmentation is achieved. The energy functional well describes the property of SAR image accurately and automatically extracts the region of interest in SAR image without any speckle pre-processing step. The functional consist of a region based term and a boundary based term. The region based term is derived from SAR image statistical property and measure the conformity of image data to a gamma model. The boundary based term is related to the edge gradient and alliance the boundary to the image pixels with maximum gradient while keeping its smoothness which describes region property and boundary property of SAR image simultaneously. Target extraction is implemented by minimizing the energy criterion via variational level set approach. The segmentation method based on this paper is more accurate than segmentation method with active contours and level sets.

\subsection{A Variational Level Set Approach to Segmentation and Bias Correction of Images with Intensity Inhomogenity [8]}

This paper presents a variational level set approach to join segmentation and bias corrections of images with intensity inhomogenity. Intensities in small local regions are separable despite of the inseparability of the intensities in the whole image caused by intensity inhomogenity. A weighted k mean clustering objective function is defined for image intensities in a neighborhood around each point, with the clusters centers having a multiplicative factor that estimate the bias with in the neighborhood. The objective function is then integrated over the entire domain and in cooperated in to a variational level set formulation. The energy minimization is performed by a level set evolution process. This method is able to estimate bias of general profiles. This method is robust to initialization and allows automatic applications. The level set formulation mainly consists of $\mathrm{N}=2$ (two phase) and $\mathrm{N}=4$ (multi phase process)By this we can reduce energy formulation and hence the segmentation is done. The advantage of this method is not sensitive to initialization and thereby allowing automatic applications. Reinitialization is also possible in this method.

\subsection{Variational and Shape Prior-based Level Set Model for Image Segmentation [9]}

Radiographic medical Images are consider were boundaries are not silent and object having the same gray level as other structure in the image, so we need a prior information about the shape that forces the level set to be closed to a signed distance function which avoids the re initialization procedure. The proposed method uses a prior information about the boundary so that the active contour or level set will evolve according to a known shape. The proposed segmentation method needs a training shape. The shape variants are computed by principle component analysis (PCA). The new shape prior is the matrix of Eigen vectors and the vector of shape parameters to be determined. A new energy functional is defined for image segmentation which is used to avoid the re initialization process and its related draw backs

\subsection{A Multiphase Level Set Framework for Image Segmentation Using the Mumford and Shah Model [10].}

This paper proposes a new multiphase level set frame work for image segmentation using the Mumford and Shah Model for piece wise constant and piece wise smooth optimal approximations. This method is a generalization of active contour model without edges based two phase segmentation in the case of piece wise smooth only two level set functions formerly suffix to represent any partition based on the four colour theorem in the piece wise constant case only log $n$ level set functions for any phases in the piece wise constant case. This avoids the problem of vaccum and overlaps and represents boundaries with complex topology including triple junction. The multiphase formulation is different than the classical approaches and has the advantages that the 
phases cannot produce vacuum or overlap by construction and it minimizes the computational cost by reducing the number of level set functions.

\section{Conclusion}

A considerable challenge in image segmentation in real world images is intensity inhomogenity. The existing image segmentation algorithms rely on region based and it typically features similarity of image intensities in the region of interest. Because of the presence of intensity non uniformity the segmentation results are not accurate. So a Variational level set model for segmentation and bias correction of images with intensity inhomogenity is presented..From all the above papers we can formulate that level set method can be performed very efficiently for the medical image segmentation. Level set method which aims on implementation of a level set approach for active contour image segmentation for MRI images. The method is well suited for MR images. The proposed method defines that in the presence of intensity inhomogenity also, segmentation can be successfully performed with accurate results

\section{References:}

[1] Tranos Zuva,Oludayo O,Olugbara,Sunday O,Ojo and Seleman Ngwira,Image Segmentation Available Techniques,Developmaents and Open Issues, Canadian Journal on Image processing and Computer Vision Vol 2 No:3 March 2011.

[2] StanleyOsher and J. Sethian, Fronts propagating with curvature-dependent speed: Algorithms based on Hamilton-Jacobi formulations, J. Comp.Phys., vol. 79, no. 1, pp. 12-49, Nov. 1988

[3] Kan Cheng, Lixu Gu, and Jianrong Xu,A Novel Shape Prior Based Level Set Method for Liver Segmentation From MR Images, Proceedings of the 5th International Conference on Information Technology and Application in Biomedicine, in conjunction with The 2nd International Symposium \& Summer School on Biomedical and Health Engineering Shenzhen, China, May 30-31, 2008

[4] Samir BARA,Mounir Ait Kerroum,Ahmed Hammouch and Driss Aboutajdine, Variational Image Segmentation Models:Application to medical images MRI, -978-1-61284-732-0/11/\$26.00 @2010 IEEE

[5] Kaihua Zhang a, Lei Zhang a, 1 and Su Zhang,A Variational Multi Phase Level Set Approach To Simultaneous Segmentation And Bias Correction

[6] Pan Lin, Chong-Xun Zheng, Yong Yang,Model-Based Medical Image Segmentation: A Level Set Approach,Proceedings of the ${ }^{\text {th }}$ World Congress on Intelligent Control and Automation. June 15-19, 2004, Hangzhou. P.R.China

[7] Zongjie Cao, Yiming Pi, Xiaobo Yang, Jintao Xiong, a Variational Level Set SAR Image Segmentation Approach Based on Statistical Model

[8] Chunming Li, RuiHuang, Zhaohua Ding, Chris Gatenby, Dimitris Metaxas,and John Gore, A Variational Level Set Approach to Segmentation and Bias Correction of Images with Intensity Inhomogeneity,D. Metaxas et al. (Eds.): MICCAI 2008, Part II, LNCS 5242, pp. 1083-1091, 2008. Springer-Verlag Berlin Heidelberg 2008

[9] El Hadji S. Diop, Silèye O. Ba, Taha Jerbi and Valérie Burdin,Variational and Shape Prior-based Level Set Model for Image Segmentation

[10] Luminita A. Vese \& Tony F. Chan, A Multiphase Level Set Framework for Image Segmentation Using the Mumford and Shah Model, International Journal of Computer Vision 50(3), 271-293, 2002@ 2002 Kluwer Academic Publishers. Manufactured in the Netherlands 